\section{PROCEEDINGS A}

royalsocietypublishing.org/journal/rspa

\section{Research}

Cite this article: Krenk S, Møller RN. 2019

Turbulent wind field representation and conditional mean-field simulation. Proc. $R$. Soc. A 475: 20180887.

http://dx.doi.org/10.1098/rspa.2018.0887

Received: 18 December 2018

Accepted: 22 February 2019

\section{Subject Areas:}

applied mechanics, computer modelling and simulation, computational mechanics

\section{Keywords:}

wind field turbulence, conditional mean simulation, generalized von Kàrmàn spectrum, stretched isotropic turbulence

\section{Author for correspondence:}

Steen Krenk

e-mail:sk@mek.dtu.dk

\section{Turbulent wind field}

representation and conditional mean-field simulation
Steen Krenk' and Randi N. Møller ${ }^{1,2}$

${ }^{1}$ Department of Mechanical Engineering, Technical University of Denmark, DK-2800 Kongens Lyngby, Denmark

${ }^{2}$ Rambøll, Hannemanns Alle, DK-2300 Copenhagen, Denmark
The covariance structure of a homogeneous isotropic turbulent wind velocity field is derived in terms of modified Bessel functions for an extended form of the Kàrmàn velocity spectrum, including explicit expressions for the transverse coherence functions. A concept of transformed isotropic turbulence is introduced to account for differences in the axial, transverse and vertical fluctuating wind velocities and length scales in natural wind. A special form of the auto-regressive simulation format is developed for convected turbulence with exponentially increasing intervals to the regression planes. In each step, the wind velocity field in a transverse plane is represented by a conditional mean field and a stochastic contribution determined explicitly by the time-space covariances. Simulation results are presented for a square area of dimension less than the integral length scale, representative of buildings and wind turbines, and a horizontal line of length six times the length scale, representative of a long-span bridge. The simulations demonstrate high accuracy of simulated spectral densities, covariance functions and transverse coherence functions. The simulated results do not show visible dependence on the specific points used for the simulated records. The efficiency and the free simulation point configuration suggest high competitiveness compared to fast Fourier transform-based spectral methods.

\section{Introduction}

Wind load constitutes an important aspect of the loading of many structures like, e.g. bridges, towers and wind turbines. With increasing structural flexibility, the 
fluctuating component of the wind load gains increased importance, and as most modern structures require analysis by numerical models, the simulation of realistic wind fields for calculation of structural loads plays a central role. Also, the requirements of the simulated wind field have increased-from the longitudinal component used with stationary structures, over the longitudinal and vertical components used, e.g. for dynamics of bridge decks, to the fully threedimensional wind fields needed for wind turbine loads. The simulation models fall largely in two categories: spectral methods, often using fast Fourier transform (FFT) techniques; and sequential models, typically of auto-regressive moving-average (ARMA) type. The wind field properties are typically given in terms of a more or less detailed spectral description, suggesting the use of FFT-based spectral methods. However, transform techniques, and in particular the FFT method, place restrictions on the spatial configuration in the form of regular intervals and rectangular grids, and furthermore the underlying discrete Fourier transform leads to periodic time histories that are not directly amenable to extension by restart of the simulation. By contrast, simulations by recurrence techniques, such as the use of ARMA processes, are computationally extremely efficient and can be continued in a fully consistent manner from a rather small amount of stored data. In the recurrence techniques, the main challenge is the development of efficient calibration techniques that select the number of matrices and their optimal calibration from the desired wind field properties.

A survey of the early development of multi-component spectral simulation, using random phase and direct factorization of the spectral density matrix, has been given by Shinozuka \& Deodatis [1]. A multi-component form was presented by Deodatis [2], in which the phase of the different components was offset by sub-increments, thereby creating a longer period of the simulated records. The Fourier technique was used to simulate a fully three-dimensional wind velocity vector field with a perturbation-based anisotropic modification of the isotropic spectral density tensor [3], by Mann \& Krenk [4,5], introducing an anti-aliasing frequency filter and Gaussian white noise amplitudes. An alternative factorization of the spectral matrix in terms of frequency-dependent eigenvectors was introduced by Li \& Kareem [6]. This format identifies the main contributing modes, and it is suitable for reduction of the number of simulated components. It has been used for simulating turbulent wind loads generated by the longitudinal component by Di Paola $[7,8]$, and for a general discussion of wind simulation techniques by Chen \& Kareem [9]. Procedures for reduction of the dimension of the independent simulation input, e.g. by proper orthogonal decomposition (POD) and Karhunen-Loève expansion techniques, have been surveyed by Solari and colleagues [10,11] and Stefanou \& Papadrakakis [12]. An interesting extension of the typical spectral procedure, in which the wind field is described in terms of convected turbulence, has been presented by Hémon \& Santi [13]. In their procedure, the wind field is represented by a combination of eigen-solutions to the spatial covariance problem as well as eigen-solutions for a temporal covariance problem.

In spite of the generality of the spectral representation and simulation methods, only $[4,5]$ use a fully consistent three-dimensional spectral density, whereas the other papers are based on a representative spectral density, e.g. of the longitudinal component, supplemented by an empirically based transverse coherence function, typically of the exponential form $\exp (-C \omega \Delta y / U)$, introduced by Davenport and discussed, e.g., in [14]. The spectrally based simulation techniques require a factorization of the spectral density matrix, typically by an eigenvector decomposition or by direct triangular matrix product decomposition. In the case of equally spaced simulation points on a line, the exponential representation of the transverse coherence offers the possibility of explicit factorization [15-17], and direct simulation of equally spaced points supplemented by a separate set of points has been considered by Peng et al. [18]. In [19], the transverse coherence function for line-based separation was used to construct a time-space-based spectral density and a corresponding FFT formulation. In the exponential coherence format, the combination of the frequency $\omega$ and the transverse distance $\Delta y$ implies that low-frequency components exhibit excessive coherence for large separation distances. Recent measurements of the transverse coherence support the lack of full coherence for low frequencies and large separation [20-22]. A resolution of this problem in terms of alternative coherence 
representations was presented in [23], based on the work of Kristensen \& Jensen [24] using the von Kàrmàn spectral density, [25].

An alternative to spectral FFT-based simulation methods is constituted by the family of sequential filter-based procedures consisting of auto-regressive (AR), moving average (MA) and the combined auto-regressive moving average (ARMA) models. The time is discretized into finite-typically equal-intervals, and each step consists of generation of a random input vector corresponding to the number of velocity components at any given time, and the formation of the new velocity values as a weighted linear combination of the simulated current white noise components, the white noise components at a set of previous times, and the wind velocities from a set of previous times. Early work on sequential stochastic field simulation was presented by Mignolet \& Spanos $[26,27]$. In this work, as well as in later works on wind field simulation, e.g. $[28,29]$, priority was given to AR and ARMA processes and calibration techniques based on underlying spectral distributions. It turns out that the coefficient matrices in auto-regressive time-space processes can be determined directly from the set of covariance matrices for the velocity components of the current and the regression steps. The key point is the availability of the full time-space covariance matrices. A simple single-step procedure for simulation of convected three-dimensional wind turbulence was presented in [30], and the present paper develops a more accurate multi-step auto-regressive format with coefficient matrices determined explicitly in terms of the two-point covariances of the convected turbulence. The wind velocity covariance is based on convected fully three-dimensional isotropic turbulence using the von Kàrmàn spectral density [25], and a simple scaling procedure is presented for adjusting the length scale and velocity intensity in the axial and transverse directions. The turbulence representation also contains explicit formulae for the transverse coherence functions, and simulation examples demonstrate that these are reproduced faithfully by the proposed method.

\section{Representation of isotropic turbulence}

The turbulent wind field used in the present paper is represented in the form of convected homogeneous isotropic incompressible turbulence as described by Batchelor [3]. The representation is for a three-dimensional homogeneous stochastic turbulent velocity field that is translated by the mean wind velocity $U$. When observing the wind field from a fixed point in space, the convected velocity field passes by at the speed $U$, thereby effecting a replacement of the along-wind coordinate by time, $x_{1}=-U t$. This leads to a compact representation in which the time-invariant spatially three-dimensional field effectively is transformed into a twodimensional time-dependent field. Clearly this representation is most suitable for scenarios where the spatial distribution is closely represented by a cross-sectional plane. It holds the advantage that turbulence can be represented as a three-dimensional isotropic spatial velocity field. However, this representation also imposes the requirement that the covariance functions of the time histories at a point lead directly to the representation of the transverse spatial structure of the field. Thus the spectral density of the time histories also defines properties like transverse coherence. A simple procedure for representing different length scales in the transverse directions or standard deviations of the transverse velocity components via 'stretched isotropic turbulence' is discussed in $\S 4$.

\section{(a) Isotropic correlation functions}

A concise summary of the representation of isotropic turbulence is now presented for a 'frozen' three-dimensional isotropic velocity field. It follows from invariance to coordinate transformations that the general form of the covariance tensor $\mathbf{R}(\mathbf{r})$ for the velocity components at two points separated by the vector $\mathbf{r}$ must be a linear combination of the unit tensor $\mathbf{I}$ and the tensor $\mathbf{r} \mathbf{r}^{\mathrm{T}}$. The classic form is

$$
\mathbf{R}(\mathbf{r})=\mathrm{E}\left[\mathbf{v}\left(\mathbf{r}_{0}+\mathbf{r}\right) \mathbf{v}\left(\mathbf{r}_{0}\right)^{\mathrm{T}}\right]=\sigma_{v}^{2}\left([f(r)-g(r)] \frac{\mathbf{r r}^{\mathrm{T}}}{\mathbf{r}^{\mathrm{T}} \mathbf{r}}+g(r) \mathbf{I}\right),
$$


where $r=|\mathbf{r}|$ is the distance between the two points, and $\sigma_{v}^{2}$ is the variance of a single component at a point. The functions $f(r)$ and $g(r)$ describe the lengthwise and transverse correlation, respectively.

At the typical wind speeds in the natural wind, the flow can be assumed to be incompressible. It then follows from the incompressibility condition $\nabla_{\mathbf{r}}^{\mathrm{T}} \mathbf{v}=0$ that the correlation functions are related by

$$
g(r)=f(r)+\frac{r}{2} \frac{\mathrm{d}}{\mathrm{d} r} f(r)=\frac{1}{2 r} \frac{\mathrm{d}}{\mathrm{d} r}\left[r^{2} f(r)\right] .
$$

Thus, the isotropic incompressible stochastic field is described entirely in terms of a single scalar correlation function, e.g. the lengthwise correlation function $f(r)$. The local incompressibility condition (2.2) can be reformulated into an integral condition. Multiplication with $r$ and integration gives

$$
\int_{0}^{\infty} g(r) r \mathrm{~d} r=\frac{1}{2}\left[r^{2} f(r)\right]_{0}^{\infty}=0,
$$

where the last equality is satisfied when the upper limit does not contribute. This relation has implications of considerable importance for the representation of correlation. If multiplied by $2 \pi$, the left side is simply the integral of the transverse correlation function $g(r)$ over an infinite plane. The result states that for any infinite plane the correlation between a normal component at a selected point and the integral of the normal component over the plane vanishes. Thus, an observation of, e.g., a positive normal component at a point does not lead to a positive expectation of the total flux through the infinite plane. This may seem obvious also for nonisotropic turbulence, but nonetheless most representations of the transverse correlation used in wind engineering violate this condition, typically by assuming the transverse correlation function $g(r)$ in the form of an exponential function. It follows from (2.3) that the transverse correlation $g(r)$ must be negative over an interval large enough to satisfy the integral condition.

\section{(b) Generalized von Kàrmàn representation}

The longitudinal and transverse correlation functions are represented via the exponential Fourier transform as

$$
\sigma_{v}^{2} f(r)=\int_{-\infty}^{\infty} F(k) \mathrm{e}^{i k r} \mathrm{~d} k \text { and } \sigma_{v}^{2} g(r)=\int_{-\infty}^{\infty} G(k) \mathrm{e}^{i k r} \mathrm{~d} k,
$$

where $k$ is the wavenumber corresponding to the spatial separation $r$. When used for convected turbulence, the 'frozen' turbulence velocity field is convected with the mean wind velocity $U$ downstream. For convection in the $x_{1}$-direction, this defines a relation between the spatial $x_{1}$ coordinate and the time $t$ of the form $x_{1}=-U t$, and thus the spatial $x_{1}$ variation corresponds to a time history and the corresponding spectral densities are easily scaled to frequency form. In the derivation of the theory and in the following simulations, the three-dimensional formulation in terms of wavenumber and coordinate components is used. Frequency and time records can then be obtained by appropriate scaling of the $k_{1}$ and $x_{1}$ components.

It is convenient to represent the spectral density function $F(k)$ in terms of the normalized form of the generalized von Kàrmàn spectral density [23],

$$
F(k)=\frac{1}{\sqrt{\pi}} \frac{\Gamma(\gamma)}{\Gamma(\gamma-1 / 2)} \frac{\sigma_{v}^{2} \ell}{\left[1+(k \ell)^{2}\right]^{\gamma}},
$$

where $\Gamma($ ) is the gamma function, and $\ell$ is a length scale. The parameter $\gamma$ controls the asymptotic behaviour of the spectral density for $(k \ell) \rightarrow \infty$. Integrability of the spectral density requires $\gamma>$ $1 / 2$. According to the Kolmogorov cascade theory, the parameter takes the value $\gamma=5 / 6$ as used in the von Kàrmàn spectral density, [25]. Here, $\gamma$ is kept as a parameter to enable a discussion of the generalized exponential format later. This form of the spectral density is analytically tractable and leads to explicit expressions for the correlation functions $f(r)$ and $g(r)$. Substitution of the 
spectral density (2.5) into (2.4a) gives

$$
f(r)=\frac{2 \ell}{\sqrt{\pi}} \frac{\Gamma(\gamma)}{\Gamma(\gamma-1 / 2)} \int_{-\infty}^{\infty} \frac{\cos (k r)}{\left[1+(k \ell)^{2}\right]^{\gamma}} \mathrm{d} k,
$$

and by 10.32 .11 [32] this determines the longitudinal correlation function as

$$
f(r)=\frac{2}{\Gamma(\gamma-1 / 2)}\left(\frac{r}{2 \ell}\right)^{\gamma-1 / 2} K_{\gamma-1 / 2}\left(\frac{r}{\ell}\right),
$$

where $K_{\gamma-1 / 2}()$ is the modified Bessel function of the second kind of order $\gamma-1 / 2$.

The transverse correlation function $g(r)$ is now conveniently found by use of the incompressibility condition (2.2). By using the differentiation formula for Bessel functions, 10.6.6 [32],

$$
\frac{\mathrm{d}}{\mathrm{d} z}\left(z^{v} K_{v}(z)\right)=-z^{v} K_{v-1}(z)
$$

with $v=\gamma-1 / 2$ it is found that

$$
\frac{r}{2} \frac{\mathrm{d}}{\mathrm{d} r} f(r)=\frac{-2}{\Gamma(\gamma-1 / 2)}\left(\frac{r}{2 \ell}\right)^{\gamma+1 / 2} K_{\gamma-3 / 2}\left(\frac{r}{\ell}\right) .
$$

Substitution of this into (2.2) then gives the corresponding transverse correlation function as

$$
g(r)=f(r)-\frac{2}{\Gamma(\gamma-1 / 2)}\left(\frac{r}{2 \ell}\right)^{\gamma+1 / 2} K_{\gamma-3 / 2}\left(\frac{r}{\ell}\right) .
$$

Thus, the two correlation functions corresponding to a velocity field with the generalized von Kàrmàn spectral density (2.5) are given by (2.7) and (2.10), respectively.

The spectral density $G(k)$ of the transverse covariance can be determined by inverting (2.4) and expressing the transverse correlation function $g(r)$ in terms of the longitudinal correlation function $f(r)$ by using (2.2). The result of this operation is

$$
G(k)=\frac{1}{2} F(k)-\frac{1}{2} k \frac{\mathrm{d}}{\mathrm{d} k} F(k) .
$$

In the particular case of the generalized von Kàrmàn spectral density (2.5), this leads to

$$
G(k)=\left(\frac{1}{2}+\frac{\gamma(k \ell)^{2}}{1+(k \ell)^{2}}\right) F(k)
$$

In the limit $k \ell \rightarrow \infty$, the asymptotic high wavenumber relation is $G(k) \simeq(\gamma+1 / 2) F(k)$.

The turbulence length scale is a representative length over which the turbulent velocity field is correlated. It is defined by

$$
\lambda=\int_{0}^{\infty} f(r) \mathrm{d} r .
$$

Substitution of the lengthwise correlation function $f(r)$ from (2.7) gives

$$
\lambda=\frac{2 \ell}{\Gamma(\gamma-1 / 2)} \int_{0}^{\infty}\left(\frac{r}{2 \ell}\right)^{\gamma-1 / 2} K_{\gamma-1 / 2}\left(\frac{r}{\ell}\right) \mathrm{d} r=\frac{\Gamma(1 / 2) \Gamma(\gamma)}{\Gamma(\gamma-1 / 2)} \ell,
$$

where the integral formula 10.43.19 [32] has been used.

\section{Transverse coherence}

The correlation function of the velocity components at two points gives a measure of statistical dependence of the fluctuations. However, more detailed information can be obtained by considering the individual spectral components associated with any particular frequency. This leads to the introduction of the coherence function of any two velocity components at two separate points. The most direct measure is the cross-spectral density normalized by the square root of the product of the spectral densities of the two velocity components. This is sometimes called the root-coherence, reserving the term coherence for the square of this quantity. However, 
in the present context, it is convenient to use the term coherence for the normalized crossspectral density. Its usefulness is closely linked to the fact that the spectral contributions at various frequencies exhibit different spatial distribution. In connection with a representation of the turbulent velocity field in the form of a convected homogeneous spatial field, only velocities at points with transverse separation retain the spatial coherence characteristics, constituting important components in the classic design of slender structures for wind loads [14].

\section{(a) A scalar potential}

When extracting the coherence from the convected velocity field, a full spectral representation of the covariance tensor is needed in the form of the three-dimensional wavenumber integral

$$
R_{i j}(\mathbf{x})=\int_{-\infty}^{\infty} \int_{-\infty}^{\infty} \int_{-\infty}^{\infty} \Phi_{i j}(\mathrm{k}) \mathrm{e}^{i \mathrm{k} \cdot \mathrm{x}} \mathrm{dk} .
$$

In the present context, the velocity field is isotropic and incompressible, and therefore of the form

$$
\Phi_{i j}(\mathrm{k})=\frac{E(k)}{4 \pi k^{4}}\left(k^{2} \delta_{i j}-k_{i} k_{j}\right),
$$

where $\delta_{i j}$ is Kronecker's delta, and $E(k)$ is the energy density in the scalar wavenumber interval $[k, k+d k]$. It turns out to be convenient to express the dependence on the individual components via spatial derivatives in the form

$$
R_{i j}(\mathbf{x})=\left(\frac{\partial^{2}}{\partial x_{i} \partial x_{j}}-\delta_{i j} \frac{\partial^{2}}{\partial x_{l} \partial x_{l}}\right) \Psi(\mathbf{x})
$$

where the scalar potential

$$
\Psi(\mathbf{x})=\int_{-\infty}^{\infty} \int_{-\infty}^{\infty} \int_{-\infty}^{\infty} \frac{E(k)}{4 \pi k^{4}} \mathrm{e}^{i k \cdot x} \mathrm{dk}
$$

is a triple integral in terms of $E(k)$.

The energy density function $E(k)$ is closely related to the one-dimensional spectral density $F(k)$ introduced in (2.4). The relation is established, as demonstrated by Batchelor [3], by expressing $F\left(k_{1}\right)$ by the inverse of (2.4), and then introducing $\sigma_{v}^{2} f\left(x_{1}\right)=R_{11}\left(x_{1}\right)$ from (3.1) and (3.2). The final result is

$$
E(k)=k^{3} \frac{\mathrm{d}}{\mathrm{d} k}\left(\frac{1}{k} \frac{\mathrm{d} F(k)}{\mathrm{d} k}\right)=\frac{4}{\sqrt{\pi}} \frac{\Gamma(\gamma+2)}{\Gamma(\gamma-1 / 2)} \frac{(k \ell)^{4} \sigma_{v}^{2} \ell}{\left[1+(k \ell)^{2}\right]^{\gamma+2}}
$$

after substitution of the generalized von Kàrmàn spectral density $F(k)$ from (2.5). Substitution of the energy density function $E(k)$ into (3.4) gives the following expression for the scalar potential:

$$
\Psi(\mathbf{x})=\frac{\sigma_{v}^{2} \ell}{\pi^{3 / 2}} \frac{\Gamma(\gamma+2)}{\Gamma(\gamma-1 / 2)} \int_{-\infty}^{\infty} \int_{-\infty}^{\infty} \int_{-\infty}^{\infty} \frac{\ell^{4}}{\left[1+(k \ell)^{2}\right]^{\gamma+2}} \mathrm{e}^{i \mathrm{k} \cdot \mathrm{x}} d \mathrm{k} .
$$

The triple integration is divided into two parts: an integration over the $k_{2} k_{3}$-plane, followed by integration over the axial wavenumber component $k_{1}$. The first integration is carried out by transformation into polar components with $k_{2} x_{2}+k_{3} x_{3}=\kappa r \cos \theta$, leading to, 10.9.1 [32],

$$
\Psi\left(x_{1}, r\right)=\sigma_{v}^{2} \ell \frac{2 \ell^{2}}{\pi^{1 / 2}} \frac{\Gamma(\gamma+2)}{\Gamma(\gamma-1 / 2)} \int_{-\infty}^{\infty} \mathrm{e}^{i k_{1} x_{1}} \mathrm{~d} k_{1} \int_{0}^{\infty} \frac{J_{0}(\kappa r) \ell^{2} \kappa}{\left[1+\left(k_{1} \ell\right)^{2}+(\kappa \ell)^{2}\right]^{\gamma+2}} \mathrm{~d} \kappa,
$$

where $J_{0}(\kappa r)$ is the Bessel function of the first kind of order zero, and $\kappa$ and $r$ are the polar radius in the transverse wavenumber plane and in the transverse physical plane, respectively. 
The two first terms in the denominator of the integrand are combined into the form $\left(\kappa_{1} \ell\right)^{2}$ by introducing the equivalent wavenumber $\kappa_{1}$ by

$$
\kappa_{1}^{2}=k_{1}^{2}+\ell^{-2} .
$$

The integral can then be evaluated by the use of the formula, 10.22.46 [32],

$$
\int_{0}^{\infty} \frac{J_{0}(a t) t}{\left[b^{2}+t^{2}\right]^{\mu+1}} \mathrm{~d} t=\frac{a^{\mu} b^{-\mu}}{2^{\mu} \Gamma(\mu+1)} K_{-\mu}(a b)
$$

when identifying the parameters $\mu=\gamma+1, t=\kappa \ell, a=r / \ell$ and $b=\kappa_{1} \ell$. This gives the scalar potential (3.7) in the form

$$
\Psi\left(x_{1}, r\right)=\frac{2}{\Gamma(\gamma)} \int_{-\infty}^{\infty} F\left(k_{1}\right) \frac{1}{\kappa_{1}^{2}}\left(\frac{\kappa_{1} r}{2}\right)^{\gamma+1} K_{\gamma+1}\left(\kappa_{1} r\right) \mathrm{e}^{i k_{1} x_{1}} \mathrm{~d} k_{1},
$$

when the longitudinal spectral density $F\left(k_{1}\right)$ is introduced from (2.5) as

$$
F\left(k_{1}\right)=\frac{1}{\sqrt{\pi}} \frac{\Gamma(\gamma)}{\Gamma(\gamma-1 / 2)} \frac{\sigma_{u}^{2} \ell}{\left(\kappa_{1} \ell\right)^{2 \gamma}},
$$

and it is used that the sign of the subscript on the modified Bessel function $K_{\gamma+1}($ ) can be changed [32].

\section{(b) Transverse coherence functions}

The transverse coherence functions now follow from differentiation of the scalar potential $\Psi(\mathbf{x})$ according to (3.3). The simplest is the coherence of the longitudinal component derived from introduction of polar coordinates in the transverse plane,

$$
R_{11}(0, r)=-\left(\frac{\partial^{2}}{\partial x_{2}^{2}}+\frac{\partial^{2}}{\partial x_{3}^{2}}\right) \Psi(0, r)=-\frac{1}{r} \frac{\partial}{\partial r}\left(r \frac{\partial}{\partial r}\right) \Psi(0, r) .
$$

It is seen from (3.10) that the radial coordinate can be combined into the non-dimensional variable $\kappa_{1} r$. It then follows from the standard differentiation formula (2.8) that the covariance can be expressed in terms of the wavenumber integral

$$
R_{11}(0, r)=\int_{-\infty}^{\infty} F\left(k_{1}\right) \psi_{11}\left(\kappa_{1} r\right) \mathrm{d} k_{1}
$$

where the transverse coherence $\psi_{11}\left(\kappa_{1} r\right)$ is obtained as

$$
\psi_{11}\left(\kappa_{1} r\right)=\frac{2}{\Gamma(\gamma)}\left[\left(\frac{\kappa_{1} r}{2}\right)^{\gamma} K_{\gamma}\left(\kappa_{1} r\right)-\left(\frac{\kappa_{1} r}{2}\right)^{\gamma+1} K_{\gamma-1}\left(\kappa_{1} r\right)\right] .
$$

It follows from the factorization, and is easily verified, that $\psi_{11}(0)=1$. The result was found in asymptotic form with $\kappa_{1} \simeq k_{1}$ and $\gamma=5 / 6$ in [24] and the present full form in [23].

Now, consider velocity components in the $x_{2}$-direction for a separation $r$ in the $x_{2}$-direction. It follows from applying the differentiation rule (3.3) to the scalar potential (3.10) that

$$
R_{22}(0, r)=\frac{2}{\Gamma(\gamma)} \int_{-\infty}^{\infty}\left(k_{1}^{2}-\frac{1}{r} \frac{\partial}{\partial r}\right) \frac{F\left(k_{1}\right)}{\kappa_{1}^{2}}\left(\frac{\kappa_{1} r}{2}\right)^{\gamma+1} K_{\gamma+1}\left(\kappa_{1} r\right) \mathrm{d} k_{1} .
$$

Application of the differentiation formula (2.8) then gives

$$
R_{22}(0, r)=\frac{2}{\Gamma(\gamma)} \int_{-\infty}^{\infty} F\left(k_{1}\right)\left[\frac{k_{1}^{2}}{\kappa_{1}^{2}}\left(\frac{\kappa_{1} r}{2}\right)^{\gamma+1} K_{\gamma+1}\left(\kappa_{1} r\right)+\frac{1}{2}\left(\frac{\kappa_{1} r}{2}\right)^{\gamma} K_{\gamma}\left(\kappa_{1} r\right)\right] \mathrm{d} k_{1} .
$$

A more compact form of the result is obtained by expressing the function $K_{\gamma+1}\left(\kappa_{1} r\right)$ in terms of the similar functions of order one and two lower,

$$
R_{22}(0, r)=\frac{2}{\Gamma(\gamma)} \int_{-\infty}^{\infty} F\left(k_{1}\right)\left[\left(\frac{1}{2}+\gamma \frac{k_{1}^{2}}{\kappa_{1}^{2}}\right)\left(\frac{\kappa_{1} r}{2}\right)^{\gamma} K_{\gamma}\left(\kappa_{1} r\right)+\frac{k_{1}^{2}}{\kappa_{1}^{2}}\left(\frac{\kappa_{1} r}{2}\right)^{\gamma+1} K_{\gamma-1}\left(\kappa_{1} r\right)\right] \mathrm{d} k_{1} .
$$


The first factor in the first term is put outside the square brackets. According to (2.12), combination of this factor with $F\left(k_{1}\right)$ gives the transverse spectral density function $G\left(k_{1}\right)$. It follows from (2.12) that the integral then is of the form

$$
R_{22}(0, r)=\int_{-\infty}^{\infty} G\left(k_{1}\right) \psi_{22}\left(\kappa_{1} r\right) \mathrm{d} k_{1}
$$

with the coherence of the transverse velocity component for transverse separation $x_{2}=r$ obtained as

$$
\psi_{22}\left(\kappa_{1} r, \kappa_{1} / k_{1}\right)=\frac{2}{\Gamma(\gamma)}\left[\left(\frac{\kappa_{1} r}{2}\right)^{\gamma} K_{\gamma}\left(\kappa_{1} r\right)+\frac{1}{\gamma+\frac{1}{2}\left(\kappa_{1} / k_{1}\right)^{2}}\left(\frac{\kappa_{1} r}{2}\right)^{\gamma+1} K_{\gamma-1}\left(\kappa_{1} r\right)\right] .
$$

It is noted that the transverse coherence function $\psi_{22}$ depends on the length scale $\ell$, not only via the variable $\kappa_{1} r$, but also by $\kappa_{1} / k_{1}$ in the factor to the second term. It is easily verified that $\psi_{22}\left(0, \kappa_{1} / k_{1}\right)=1$. The asymptotic result for small separation corresponds to $\ell \rightarrow \infty$, whereby the factor to the second term becomes $\left(\frac{1}{2}+\gamma\right)^{-1}$. For $\gamma=5 / 6$, this reproduces the asymptotic result with factor $3 / 4$ from [24].

When denoting the separation in the $x_{2}$ direction $r$, the covariance matrix component $R_{33}(0, r)$ follows from the scalar potential (3.10) and the differentiation formula (3.3) as

$$
R_{33}(0, r)=\frac{2}{\Gamma(\gamma)} \int_{-\infty}^{\infty}\left[k_{1}^{2}-\left(1+r \frac{\partial}{\partial r}\right)\left(\frac{1}{r} \frac{\partial}{\partial r}\right)\right] \frac{F\left(k_{1}\right)}{\kappa_{1}^{2}}\left(\frac{\kappa_{1} r}{2}\right)^{\gamma+1} K_{\gamma+1}\left(\kappa_{1} r\right) \mathrm{d} k_{1} .
$$

When comparing with the similar integral for the covariance $R_{22}(0, r)$ in $(3.15)$ it is seen that only the last term containing the second derivative has been added. This term leads to a modification of the factor on the second term in (3.17). The coherence function $\psi_{33}$ is defined by normalization with respect to the transverse covariance spectrum $G\left(k_{1}\right)$, leading to

$$
\psi_{33}\left(\kappa_{1} r, \kappa_{1} / k_{1}\right)=\frac{2}{\Gamma(\gamma)}\left[\left(\frac{\kappa_{1} r}{2}\right)^{\gamma} K_{\gamma}\left(\kappa_{1} r\right)+\frac{1-\left(\kappa_{1} / k_{1}\right)^{2}}{\gamma+\frac{1}{2}\left(\kappa_{1} / k_{1}\right)^{2}}\left(\frac{\kappa_{1} r}{2}\right)^{\gamma+1} K_{\gamma-1}\left(\kappa_{1} r\right)\right] .
$$

In the asymptotic solution with $\kappa_{1} \simeq k_{1}$ the second term vanishes, and for $\gamma=5 / 6$ the result from [24] is recovered.

The covariances $R_{13}(0, r)$ and $R_{23}(0, r)$ vanish for all values of the separation distance $r$, as seen from the differentiation formula (3.3), by which the integrand from (3.4) vanishes identically. Thus, also the corresponding coherence functions $\psi_{13}\left(k_{1} r\right)$ and $\psi_{23}\left(k_{1} r\right)$ vanish. The covariance $R_{12}(0, r)$ also vanishes. In this case, the integrand does not vanish, but by the differentiation formula (3.3) it contains the factor $i k_{1}$, making the general integral a sine-transform with factor $\sin \left(k_{1} x_{1}\right)$. For $x=0$, this introduces a factor zero, so although there is an amplitude function that can be subjected to a formal normalization, the corresponding coherence function $\psi_{12}\left(k_{1} r\right)$ vanishes in the transverse plane $x_{1}=0$.

The transverse coherence functions $\psi_{11}\left(k_{1} r\right), \psi_{22}\left(k_{1} r\right)$ and $\psi_{33}\left(k_{1} r\right)$ are shown in figure 1 for the parameter value $\gamma=5 / 6$, representing the Kolmogorov high-frequency behaviour of the spectral density. When considering convected turbulence with mean velocity $U$, the argument of the covariance functions is $\omega r / U$. There are two sets of curves, one corresponding to a finite length scale $\ell$ in the von Kàrmàn spectral density and separation $r=\lambda$, and the other set representing the asymptotic case of $\kappa_{1} \simeq k_{1}$. It is observed that the asymptotic theory gives full correlation at $r=0$, whereas the finite length $\ell$ in the spectral density leads to a notable reduction of the correlation functions for $k_{1} r \lesssim 2$ for the present separation $r=\lambda$. Recent measurements in turbulent wind [20-22] confirm the general features illustrated in figure 1 .

\section{Transformed isotropic turbulence}

Natural turbulent wind fields are characterized by different turbulence intensities and different turbulence length scales in the along-wind, transverse and vertical directions. These anisotropic 


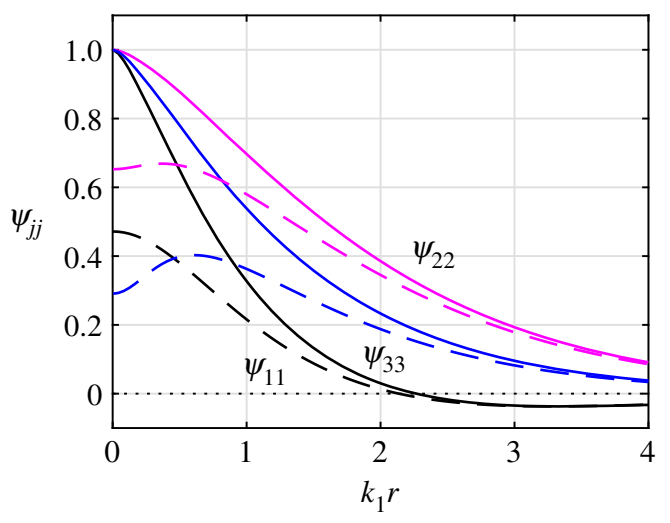

Figure 1. Transverse coherence functions $\psi_{11}\left(k_{1} r\right), \psi_{22}\left(k_{1} r\right), \psi_{33}\left(k_{1} r\right)$. Full lines $\kappa_{1}=k_{1}$, dashed lines $r=\lambda$. (Online version in colour.)

characteristics are different from those developing physically from an isotropic turbulent field, typically analysed as a perturbation building up over time.

The turbulent velocity field representation in $\$ 2$ is a special case, valid for incompressible isotropic flow. The representation in terms of the two scalar potential functions $f(r)$ and $g(r)$ follows from invariance requirements due to isotropy, and the elimination of $g(r)$ is a consequence of incompressibility. A quite general incompressible anisotropic flow field can be constructed from the three vector components of an incompressible Helmholtz potential, in which there are three intensities and each of these can be given a spatial distribution in terms of scaled coordinates. A more specialized anisotropic turbulence format is that of transformed isotropic turbulence, in which the velocity components $\mathbf{v}^{\prime}$ and corresponding spatial coordinates $\mathbf{r}^{\prime}$ are obtained from an isotropic turbulent field by a common transformation matrix $\mathbf{F}$,

$$
\mathbf{v}^{\prime}=\mathbf{F v} \quad \text { and } \quad \mathbf{r}^{\prime}=\mathbf{F r} .
$$

It follows from the differential increment relation $d \mathbf{v}^{\prime}=\left(\partial \mathbf{v}^{\prime} / \partial \mathbf{r}^{\prime}\right) d \mathbf{r}^{\prime}$ that the gradient tensor of the transformed field is

$$
\frac{\partial \mathbf{v}^{\prime}}{\partial \mathbf{r}^{\prime}}=\mathbf{F} \frac{\partial \mathbf{v}}{\partial \mathbf{r}} \mathbf{F}^{-1}
$$

The divergence is given by the trace of the gradient tensor, and when combining the factors $\mathbf{F}$ and $\mathbf{F}^{-1}$ it follows that transformation of an incompressible turbulent field by linear relations of the form (4.1) leads to an incompressible transformed field.

The simple concept of transformed isotropic turbulence enables an approximate representation of the often observed fact in turbulent wind fields that the length scales associated with alongwind, transverse and vertical separation typically satisfy the relations $\lambda_{x}>\lambda_{y}>\lambda_{z}$, with the associated standard deviations of the fluctuating wind velocity components satisfying the similar inequalities $\sigma_{u}>\sigma_{v}>\sigma_{w}$. If selecting $\sigma_{u}$ to represent the along-wind component intensity and $\lambda_{x}$, the length scale associated with the measured spectral density, there is one free parameter remaining for each of the transverse directions. This enables, by a suitable compromise, to represent the frequency spectrum together with a reduced transverse length scale and turbulence intensity. The effect of the difference in along-wind and transverse length scales and turbulence intensities has been demonstrated for wind loads and response of long-span bridges in [31], using the simulation method developed in the following.

\section{Conditional mean-field simulation}

The simulation procedure is closely related to the concept of convected turbulence and is illustrated in figure 2, showing a stack of planes parallel to the $y z$-plane. The fluctuating wind 


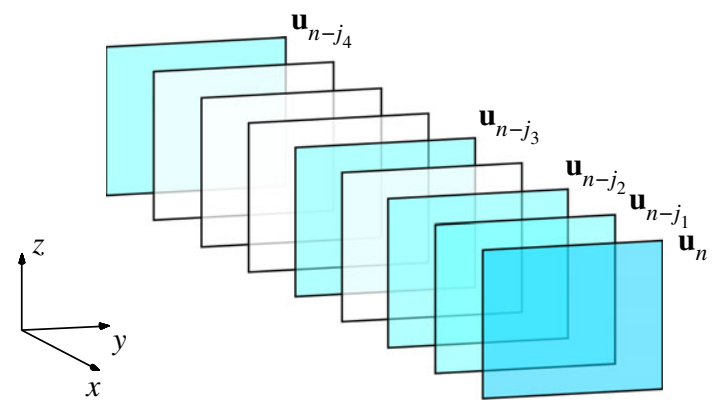

Figure 2. Conditional mean-field simulation procedure. (Online version in colour.)

velocity is represented at a set of fixed points $\mathbf{r}_{j}$ in the $y x$-plane represented by the global vector

$$
\mathbf{x}^{\mathrm{T}}=\left[\mathbf{r}_{1}^{\mathrm{T}}, \mathbf{r}_{2}^{\mathrm{T}}, \ldots, \mathbf{r}_{m}^{\mathrm{T}}\right] .
$$

The planes have the distance $\Delta x=U \Delta t$, and in a generic plane with index $n$ the velocity vectors $\mathbf{v}_{j}$ from the individual points are collected in the global vector

$$
\mathbf{u}_{n}^{\mathrm{T}}=\left[\mathbf{v}_{1}^{\mathrm{T}}, \mathbf{v}_{2}^{\mathrm{T}}, \ldots, \mathbf{v}_{m}^{\mathrm{T}}\right]_{n} .
$$

At each step of the simulation algorithm, the index $n$ is increased by one, corresponding to an increment $\Delta x$, and thus the simulation moves along the $x$-axis shown in figure 2 .

The standard format of an auto-regressive vector process is

$$
\mathbf{u}_{n}=\mathbf{A}_{1} \mathbf{u}_{n-1}+\mathbf{A}_{2} \mathbf{u}_{n-2}+\cdots+\mathbf{A}_{j} \mathbf{u}_{n-j}+\mathbf{B} \xi_{n}, \quad n=1,2, \ldots
$$

where $\mathbf{u}_{n}$ is an array containing the new set of field variables, expressed as the sum of $j$ previous sets $\mathbf{u}_{n-1}, \ldots, \mathbf{u}_{n-j}$ plus the set $\boldsymbol{\xi}_{n}$ of random variables. The random vectors $\boldsymbol{\xi}_{n}$ are here taken in the form of uncorrelated normalized normal components, whereby

$$
\mathrm{E}\left[\boldsymbol{\xi}_{i} \xi_{j}^{\mathrm{T}}\right]=\delta_{i j} \mathbf{I} .
$$

When considering the process as a development in time, the time increment $\Delta t$ between consecutive sets $\mathbf{u}_{n}$ defines the time discretization, and the depth of the filter generated by the $j$-term average defines the time interval $j \Delta t$, available for shaping the spectral properties of the process. As it turns out in the context of wind field simulation, a considerable gain in the efficiency of the auto-regressive process model is attained by selecting the distance between the previous steps to be increasing, thereby creating a longer filter length for a given number $j$ of previous values of the field variables. With this modification, the process takes the form

$$
\mathbf{u}_{n}=\mathbf{A}_{1} \mathbf{u}_{n-i_{1}}+\mathbf{A}_{2} \mathbf{u}_{n-i_{2}}+\cdots+\mathbf{A}_{j} \mathbf{u}_{n-i_{j}}+\mathbf{B} \xi_{n}, \quad n=1,2, \ldots
$$

The particular case $i_{i}=1,2,4,8$ is illustrated in figure 2 .

It is convenient to write the modified auto-regressive recurrence relation (5.5) in the form

$$
\mathbf{u}_{n}=\mathbf{A} \mathbf{w}_{n}+\mathbf{B} \boldsymbol{\xi}_{n}, \quad n=1,2, \ldots
$$

in terms of the compound matrix and vector

$$
\mathbf{A}=\left[\mathbf{A}_{1}, \ldots, \mathbf{A}_{j}\right] \text { and } \mathbf{w}_{n}^{\mathrm{T}}=\left[\mathbf{u}_{n-i_{1}}^{\mathrm{T}}, \ldots, \mathbf{u}_{n-i_{j}}^{\mathrm{T}}\right] .
$$

The matrices $\mathbf{A}$ and $\mathbf{B}$ are now determined from the covariance properties of $\mathbf{u}_{n}$ and $\mathbf{w}_{n}$. For this purpose, the following notation for covariance matrices is introduced:

$$
\mathbf{C}_{\mathbf{u u}}=\mathrm{E}\left[\mathbf{u}_{n} \mathbf{u}_{n}^{\mathrm{T}}\right], \quad \mathbf{C}_{\mathbf{u w}}=\mathrm{E}\left[\mathbf{u}_{n} \mathbf{w}_{n}^{\mathrm{T}}\right] \quad \text { and } \quad \mathbf{C}_{\mathbf{w w}}=\mathrm{E}\left[\mathbf{w}_{n} \mathbf{w}_{n}^{\mathrm{T}}\right] .
$$


It is observed that these covariance matrices are independent of the subscript $n$ for stationary fields, and for the turbulent wind field in question they are given explicitly in terms of the velocity component covariance functions in $\S 2$.

First, the matrix $\mathbf{A}$ is determined from (5.6) by observing that $\boldsymbol{\xi}_{n}$ is independent of $\mathbf{w}_{n}$, and post-multiplication by $\mathbf{w}_{n}^{\mathrm{T}}$ leads to the following equation:

$$
\mathrm{C}_{\mathrm{uw}}=\mathrm{AC}_{\mathrm{ww}} \text {. }
$$

Post-multiplication of this equation with the inverse of $\mathbf{C}_{\mathbf{w w}}$ gives the explicit expression for the compound regression matrix $\mathbf{A}$,

$$
A=C_{u w} C_{w w}^{-1} .
$$

When there are $j$ regression terms, the size of $\mathbf{w}_{n}$ is $j$ times larger than that of $\mathbf{u}_{n}$, and thus it may be numerically advantageous to avoid forming the inverse matrix and simply solve the equation system arising from taking the transpose of (5.9),

$$
\mathbf{C}_{\mathbf{w w}} \mathbf{A}^{\mathrm{T}}=\mathbf{C}_{\mathbf{u w}}^{\mathrm{T}} .
$$

It is observed that the conditional expectation of $\mathbf{u}_{n}$ for given $\mathbf{w}_{n}$ follows from (5.6) as

$$
\mathrm{E}\left[\mathbf{u}_{n} \mid \mathbf{w}_{n}\right]=\mathbf{A} \mathbf{w}_{n},
$$

and thus the auto-regressive format (5.6) defines the new variable $\mathbf{u}_{n}$ as the conditional mean value for the given regression variables $\mathbf{w}_{n}$, plus a zero-mean stochastic term $\mathbf{B} \boldsymbol{\xi}_{n}$.

The matrix $\mathbf{B}$ that distributes the stochastic input is determined from the regression equation (5.6) by moving the term $\mathbf{A} \mathbf{w}_{n}$ to the left side of the equation and multiplying each side from the right by its transpose,

$$
\left[\mathbf{u}_{n}-\mathbf{A} \mathbf{w}_{n}\right]\left[\mathbf{u}_{n}-\mathbf{A} \mathbf{w}_{n}\right]^{\mathrm{T}}=\mathbf{B} \boldsymbol{\xi}_{n} \boldsymbol{\xi}_{n}^{\mathrm{T}} \mathbf{B}^{\mathrm{T}} .
$$

When taking the expectation of this equation, using that the components of $\xi_{n}$ are uncorrelated and normalized, and substituting the regression matrix $\mathbf{A}$ from (5.10), the following equation is obtained

$$
B B^{T}=C_{u u}-C_{u w} C_{w w}^{-1} C_{w u} .
$$

Recalling the remarks concerning the undesirability of evaluating the inverse matrix $\mathbf{C}_{\mathbf{w w}}^{-1}$ in connection with the evaluation of the regression matrix $\mathbf{A}$, the equation is reformulated by introducing the matrix A from (5.10), yielding the more convenient expression

$$
\mathbf{B B}^{\mathrm{T}}=\mathbf{C}_{\mathbf{u u}}-\mathbf{A C}_{\mathbf{w w}} \mathbf{A}^{\mathrm{T}} \text {. }
$$

The solution can be obtained either by factorization into triangular matrices or by an eigenvalue analysis.

The expression on the right side of (5.13) is recognized as the conditional variance of $\mathbf{u}_{n}$ for given $\mathbf{w}_{n}$, whereby

$$
\operatorname{Cov}\left[\mathbf{u}_{n}, \mathbf{u}_{n} \mid \mathbf{w}_{n}\right]=\mathbf{B B}^{\mathrm{T}} .
$$

This formula and the corresponding conditional mean formula (5.12) assign specific roles to the matrices $\mathbf{A}$ and $\mathbf{B}$ : the recurrence matrix $\mathbf{A}$ defines the conditional mean field, and the matrix product $\mathbf{B B}^{\mathrm{T}}$ determines the intensity and correlation of the fluctuations around this mean.

\section{Wind field simulation}

An application area of considerable current interest is that of turbulent wind load on structures. Specific applications range from structures like towers, high-rise buildings and long-span bridges to wind turbines. Traditionally, the level of ambition regarding the representation of the turbulent wind load in these applications has been very different, with structural applications often using simple analytical approximations for the resulting load, whereas wind turbine design typically uses rather detailed wind field simulation, often based on FFT techniques, see e.g. [4,5]. A field of growing interest is the representation of wind loads on long-span bridges with lengths 
of up to $2000-3000 \mathrm{~m}$. These applications require simulations of very long and narrow areas, not immediately suitable for frequency representation and Fourier methods. Furthermore, these applications require a realistic representation of the transverse length scale as considerably shorter than the equivalent axial length scale. This can be accomplished by suitable stretching as described in $\S 4$ and illustrated in [31]. As the stretched field can be generated directly from a corresponding isotropic field, the following simulations are limited to isotropic fields.

\section{(a) Isotropic wind field correlation}

The simulations are performed by using the recurrence relation (5.5) in which the compound recurrence matrix $\mathbf{A}$ and the input matrix $\mathbf{B}$ are evaluated from the correlation matrices for the points at all the involved cross-sections by (5.11) and (5.15). The correlation matrix for two crosssections with distance $j$ is of the form

$$
\mathbf{C}_{j}=\mathrm{E}\left[\mathbf{u}_{n} \mathbf{u}_{n-j}^{\mathrm{T}}\right]=\left[\begin{array}{ccc}
\vdots & \vdots & \vdots \\
\vdots & \mathrm{E}\left[\mathbf{v}_{p, n} \mathbf{v}_{q, n-j}^{\mathrm{T}}\right] & \vdots \\
\vdots & \vdots & \vdots
\end{array}\right]=\left[\begin{array}{ccc}
\vdots & \vdots & \vdots \\
\vdots & \mathbf{R}\left(\mathbf{r}_{p, n}-\mathbf{r}_{q, n-j}\right) & \vdots \\
\vdots & \vdots & \vdots
\end{array}\right] .
$$

where the subscripts $p$ and $q$ identify the points in the two cross-sections. The final form consists of the $3 \times 3$ block matrices representing the covariance between the point $\mathbf{r}_{p}$ in layer $n$ and the point $\mathbf{r}_{q}$ in layer $n-j$.

For an isotropic field, the covariance function is given in terms of the axial and transverse correlation functions $f(r)$ and $g(r)$ by (2.1). According to Kolmogorov's cascade theory, the highfrequency behaviour of the spectral density gives $\gamma=5 / 6$, and the axial and transverse correlation functions then follow from (2.7) and (2.10) as

$$
f(r)=\frac{2}{\Gamma(1 / 3)}\left(\frac{r}{2 \ell}\right)^{1 / 3} K_{1 / 3}\left(\frac{r}{\ell}\right) \text { and } g(r)=f(r)-\frac{2}{\Gamma(1 / 3)}\left(\frac{r}{2 \ell}\right)^{4 / 3} K_{-2 / 3}\left(\frac{r}{\ell}\right)
$$

with the length parameter $\ell$ calculated from (2.14) as $\ell=1.339 \lambda$. For a detailed analysis of the simulated wind field, the transverse coherence functions are available from $\S 3$ in terms of $K_{5 / 6}\left(\kappa_{1} r\right)$ and $K_{-1 / 6}\left(\kappa_{1} r\right)$.

If only considering the simulation, the axial and transverse correlation functions $f(r)$ and $g(r)$ can alternatively be expressed in terms of Airy functions [32]. This formulation uses a non-dimensional transformed variable $z$ to represent the distance,

$$
z=\left(\frac{3 r}{2 \ell}\right)^{2 / 3},
$$

whereby

$$
f(r)=\frac{\operatorname{Ai}(z)}{\operatorname{Ai}(0)} \quad \text { and } \quad g(r)=f(r)+\frac{z}{3} \frac{\operatorname{Ai}^{\prime}(z)}{\operatorname{Ai}(0)} .
$$

Here, $\operatorname{Ai}(z)$ is the Airy function and $\operatorname{Ai}^{\prime}(z)$ its derivative. Like the modified Bessel functions $K_{v}(r / \ell)$, the Airy function $\operatorname{Ai}(z)$ and its derivative $\operatorname{Ai}^{\prime}(z)$ are directly available in several programming environments.

A simple approximate formulation of the spatial correlations can be obtained by using the exponent $\gamma=1$, whereby

$$
f(r)=\mathrm{e}^{-r / \lambda} \quad \text { and } \quad g(r)=\left(1-\frac{1}{2} \frac{r}{\lambda}\right) \mathrm{e}^{-r / \lambda}
$$

This formulation improves on the classic exponential engineering approximation by retaining the integral zero mean property (2.3). A simple exponential format may also be obtained for the transverse coherence functions by selecting $\gamma=1 / 2$, [23], but it is noted that this value represents the limit to a non-integrable spectral density (2.5). The resulting exponential coherence format has recently been compared with measured records in [21] and [22], confirming the general feature of 
(a)

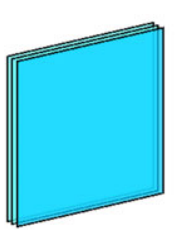

(b)

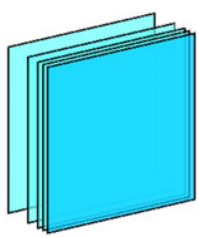

(c)

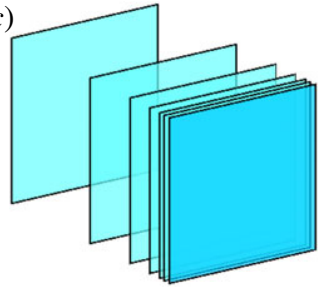

Figure 3. Regression planes, $(a) j=1,2,(b) j=1,2,4,8,(c) j=1,2,4,8,16,32$. (Online version in colour.)

Table 1. Simulation parameters: square field. Lengths in metres.

$\begin{array}{llllllllll}\sigma_{u} & \lambda & L_{y} & L_{z} & \Delta x & \Delta y & \Delta z & N_{x} & N_{y} & N_{z} \\ 1.0 & 300 & 100 & 100 & 5.0 & 5.0 & 5.0 & 10^{6} & 21 & 21\end{array}$

the $\kappa_{1} r$ dependence. In view of the limit on the general consistency of the exponential coherence format, these results deserve a comparison with the consistent coherence functions from $\S 3$ with $\gamma=5 / 6$.

\section{(b) Square cross-section field}

First an isotropic wind field with square cross-section area of dimension $L_{y}=L_{z}=100 \mathrm{~m}$ is considered. The length scale is $\lambda=300 \mathrm{~m}$, and the spatial increments are taken to be equal, $\Delta x=\Delta y=\Delta z=5.0 \mathrm{~m}$. This corresponds to the along-wind resolution of $\lambda / \Delta x=60$, and crosssection resolution $L_{y} / \Delta y=L_{z} / \Delta z=20$. The record length is $N_{x}=10^{6}$ steps, and the standard deviation of the velocity components is set to $\sigma_{u}=1.0 \mathrm{~m} \mathrm{~s}^{-1}$, but appears only as a numerical scaling factor. The data, representative of e.g. a large wind turbine, are summarized in table 1.

In addition to the parameters in table 1, the simulation process also depends on the number and distribution of the regression terms. It turns out that a distribution of the regression intervals according to $j=2^{i-1}, i=1,2, \ldots$ is quite effective. The cases with 2,4 and 6 terms are illustrated in figure 3 . The axes are defined as shown in figure 2 . With the parameters from table 1 , the intervals spanned by the regression terms are $0.033 \lambda, 0.133 \lambda$ and $0.533 \lambda$.

In many applications, such as e.g. response analysis of structures exposed to the wind field, the spectral density plays a central role, and thus the ability to reproduce the spectral density accurately in the simulated wind field is important. Here, the spectral density of the along-wind velocity component $u$ at the centre of the cross-section square is chosen to illustrate the spectral characteristics of the simulated field. The results from the three sets of recurrence intervals just discussed are shown in figure $4 a, c, e$ using the standard non-dimensional spectral density $k_{1} S_{u u}\left(k_{1}\right) / \sigma_{u}^{2}$ in terms of the along-wind wavenumber $k_{1}$ in a double-logarithmic plot. It is seen that the simple two-step memory underestimates the maximum of the spectral density in the interval $k_{1} \simeq 0.005-0.01$. This problem is nearly removed when using four regression planes, and finally a full representation is obtained with six regression planes. The right column contains the similar simulation results obtained by including the full set of regression steps with equal spacing $\Delta x$. The results are remarkably similar to those obtained by fewer steps with increasing intervals in the left column. Thus there is no visible improvement by including all the regression steps with interval $\Delta x$. All the simulated results show a slight overestimation of the spectral density for the largest wave numbers.

The results in figure 4 show that a quite accurate representation of the auto-spectral density of the along-wind component at the centre simulation point is obtained for the set of regression intervals $j=1,2,4,8,16,32$. This set of regression intervals is now used to simulate a wind field where all three wind velocity components are sampled at three points as illustrated in figure $5 a$, a 
(a)

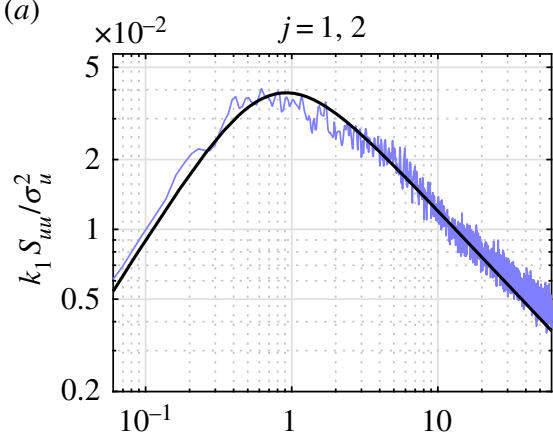

(c)

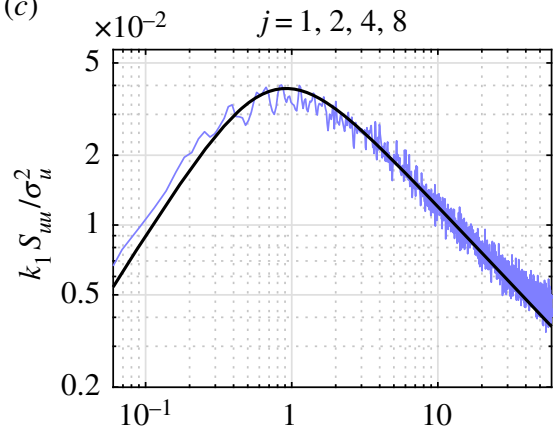

(e)

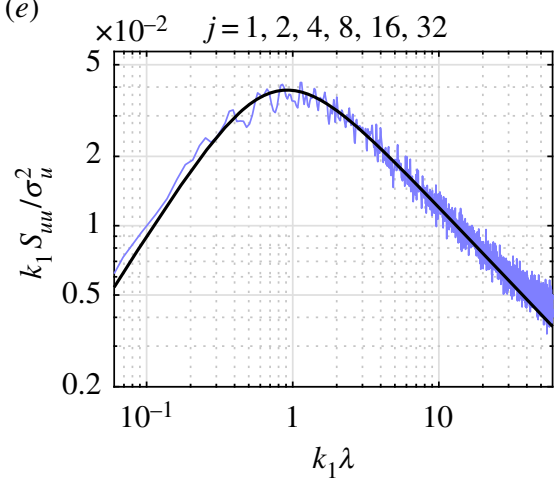

(b)

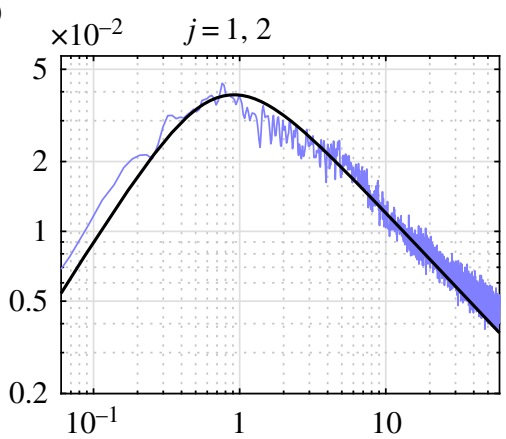

(d)

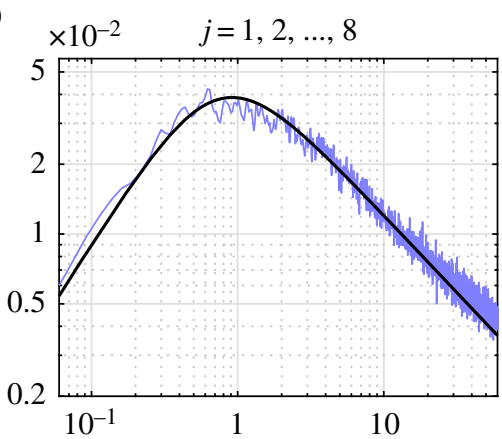

$(f)$

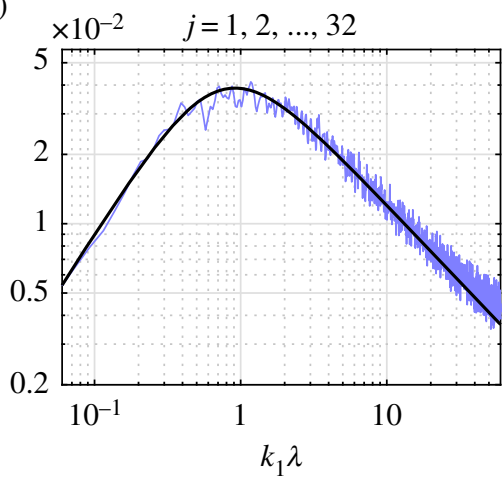

Figure 4. Estimated auto-spectral densities (blue line), target spectrum (black line). ( $a, c, e)$ Left column: exponentially spaced memory steps; $(b, d, f)$ right column: full set of equally spaced memory steps. (Online version in colour.)

corner, a side centre and the centre node. The spectral densities $k_{1} S_{u u}\left(k_{1}\right), k_{1} S_{v v}\left(k_{1}\right)$ and $k_{1} S_{w w}\left(k_{1}\right)$ are shown in figure $5 b-d$, respectively. Each figure contains the simulated results at each of the three points, together with the relevant theoretical spectral density $F\left(k_{1}\right)$ from (3.11) or $G\left(k_{1}\right)$ from (2.12). The quality of the simulated results appears to be similar for each of the velocity components, and there is no notable difference between the records at the three points. Thus, the simulated results seem unbiased by the location of the sampling point and do not exhibit any boundary effects from the simulated cross-section domain.

The results in figures 4 and 5 have addressed properties of the individual simulated time records. In addition, there is the correlation properties between velocity components at points in the simulated cross-sections. Figure $6 a$ shows the horizontal and vertical lines of points through the centre, and the correlations $C_{u u} / \sigma_{u}^{2}, C_{v v} / \sigma_{v}^{2}$ and $C_{w w} / \sigma_{w}^{2}$ evaluated from the simulated records along these lines. Figure $6 b$ shows the correlation of the along-wind component $u$ together with 
(a)
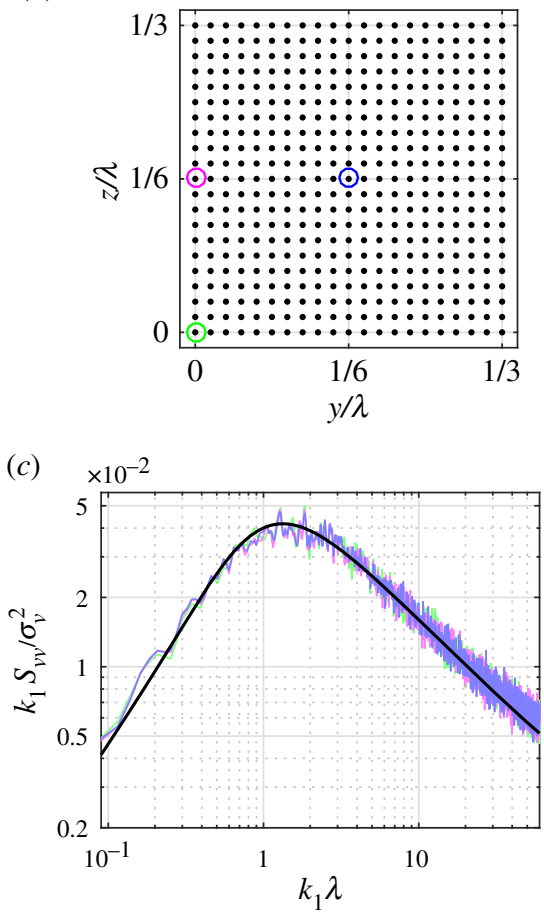

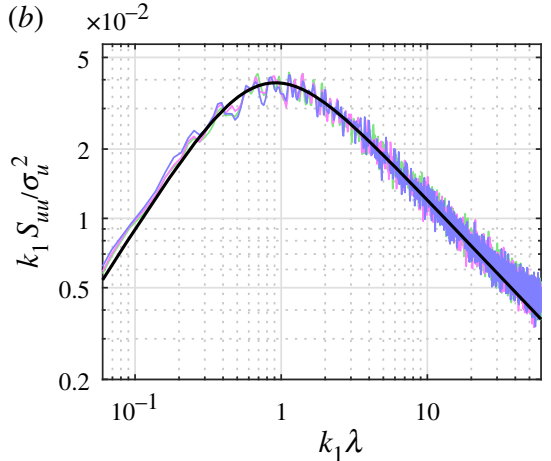

(d)

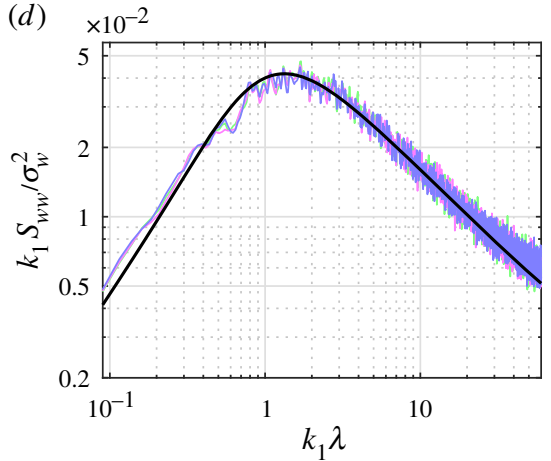

Figure 5. Target spectrum (black line), estimated auto-spectral densities in thin line (blue, magenta and green). (0nline version in colour.)

(a)

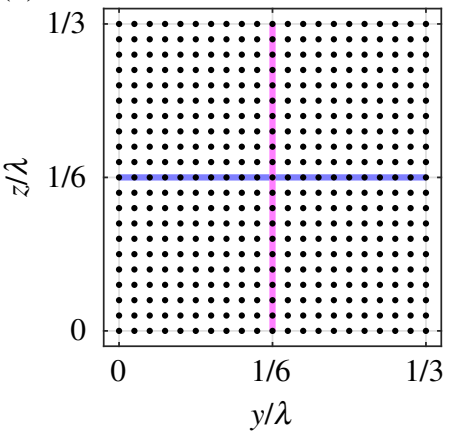

(c)

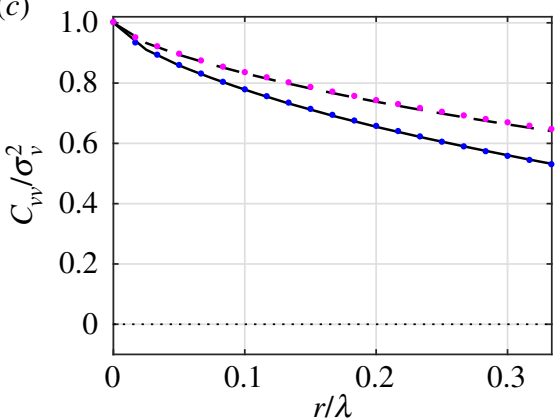

(b)

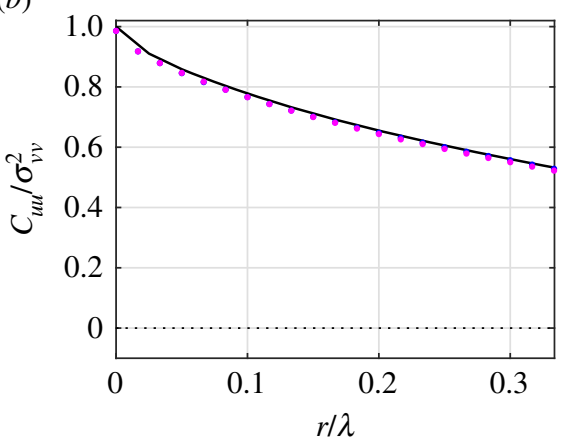

(d)

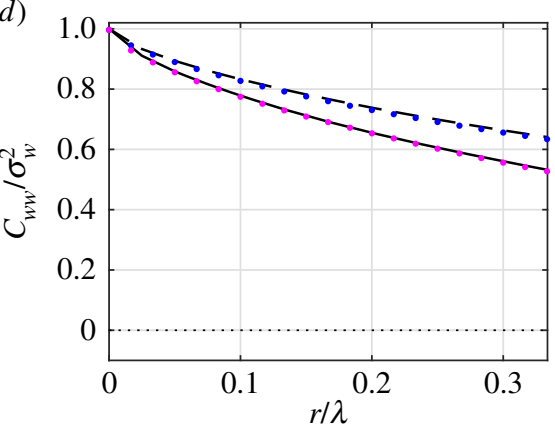

Figure 6. Theoretical transverse correlation $g(r)$ (full line) and axial correlation $f(r)$ (dashed line), estimated correlations (blue dotted, magenta dotted). (Online version in colour.) 

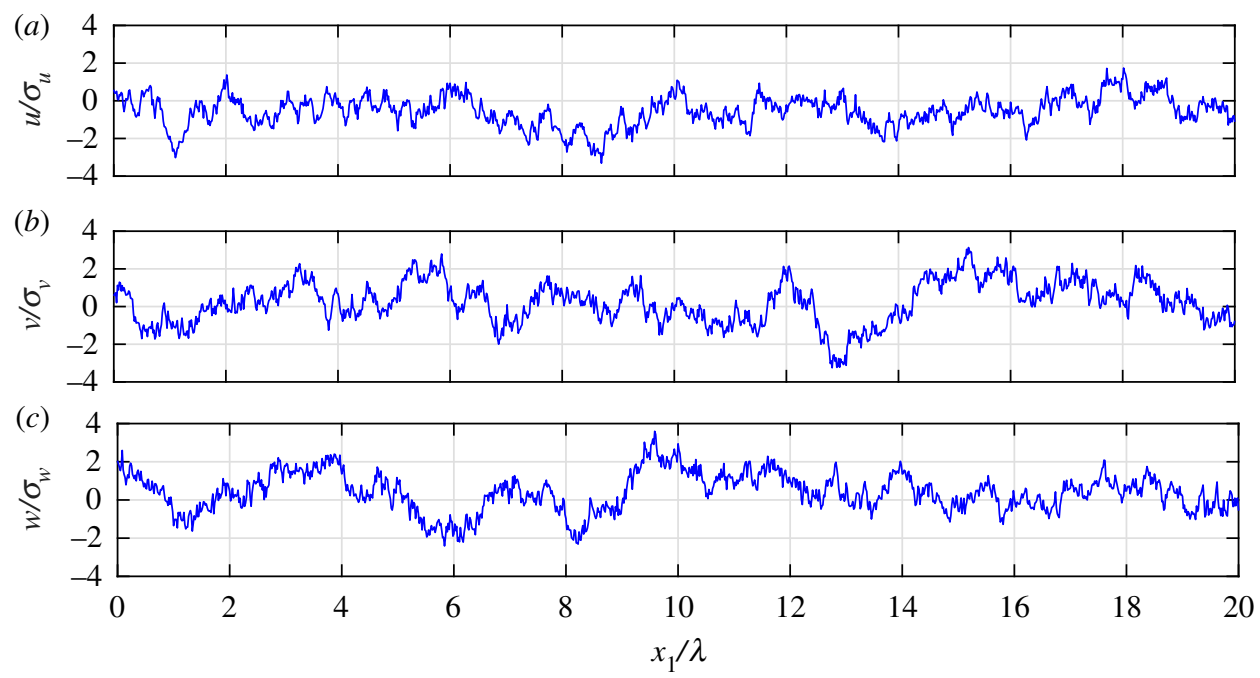

Figure 7. Equivalent time records of turbulent velocity components, $x_{1} / \lambda=(U / \lambda) t$. (Online version in colour.)

Table 2. Simulation parameters: horizontal line field. Lengths in metres.

$\begin{array}{cccccccccc}\sigma_{u} & \lambda & L_{y} & L_{z} & \Delta x & \Delta y & \Delta z & N_{x} & N_{y} & N_{z} \\ 1.0 & 300 & 1800 & - & 5.0 & 9.0 & - & 10^{6} & 201 & 1\end{array}$

the theoretical value $g(r)$ from $(6.2 \mathrm{~b})$ along the horizontal and vertical lines. The simulated results are indistinguishable and extremely close to the theoretical value. The correlation of the horizontal velocity component $v$ is shown in figure $6 c$ together with the theoretical value $f(r)$ from (6.2)a along the horizontal line and $g(r)$ along the vertical line. The agreement between simulated and theoretical results is very good. Finally, the similar results are shown for the vertical velocity component $w$ in figure $6 d$. It is noted that the spatial correlations are significant over the whole area as the dimensions are less than the correlation length $\lambda$.

Figure 7 shows corresponding time records of the three wind components $u, v$ and $w$ at the centre point of the simulation grid. The time is represented via the non-dimensional equivalent time $x_{1} / \lambda=(U / \lambda) t$. The scale of the actual time depends on the mean wind speed $U$ and the turbulence length scale $\lambda$. In the present case $\lambda=300 \mathrm{~m}$, and thus for a mean wind speed of $U=15 \mathrm{~m} \mathrm{~s}^{-1}$ a unit on the time axis corresponds to $\lambda / U=20 \mathrm{~s}$.

\section{(c) Horizontal line field}

The present simulation scenario is representative of the turbulent wind field on a long-span bridge. The transverse length is $L_{y}=1800 \mathrm{~m}$ and in order to test the capability of the simulation method the domain is taken as a single row of $N_{y}=201$ points, corresponding to $N_{z}=1$ and $L_{z}=0 \mathrm{~m}$. The spectral properties of the wind are the same as in the previous example with $\sigma_{u}=1.0 \mathrm{~m} \mathrm{~s}^{-1}$ and the axial integral length scale $\lambda=300 \mathrm{~m}$. The parameters are summarized in table 2 .

It was demonstrated in the previous example that an exponentially increasing distance to the regression planes in the simulation model leads to an efficient and accurate algorithm. In view of the much larger transverse dimension of the present model, $1800 \mathrm{~m}$ against $100 \mathrm{~m}$, the need for additional depth of the memory is investigated. The general exponential representation $j=m^{i-1}$ with $i=1,2, \ldots, 5$ is investigated with basis $m=2,3,4$, respectively. The simulated results are illustrated by their axial spectral density in figure 8 . The figures to the left illustrate the regression 
(a)

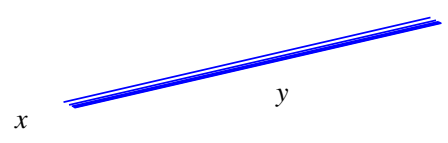

(c)

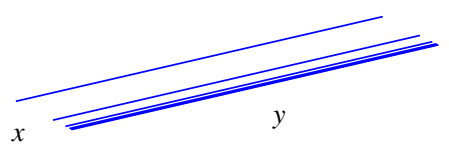

(e)

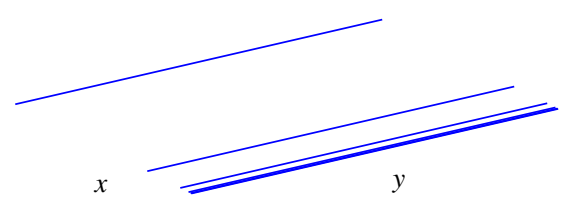

(b) $\quad \times 10^{-2} \quad j=1,2,4,8,16$

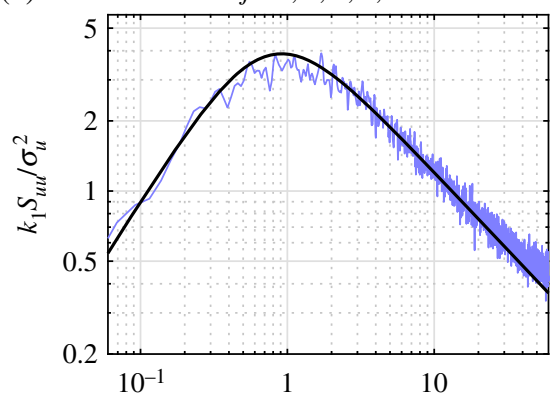

(d) $\quad \times 10^{-2} \quad j=1,3,9,27,81$

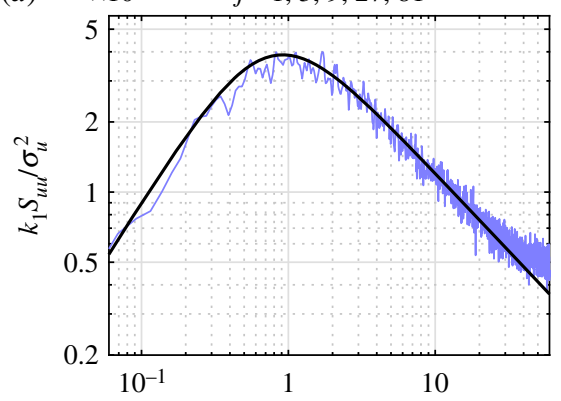

(f) $\quad \times 10^{-2} \quad j=1,4,16,64,256$

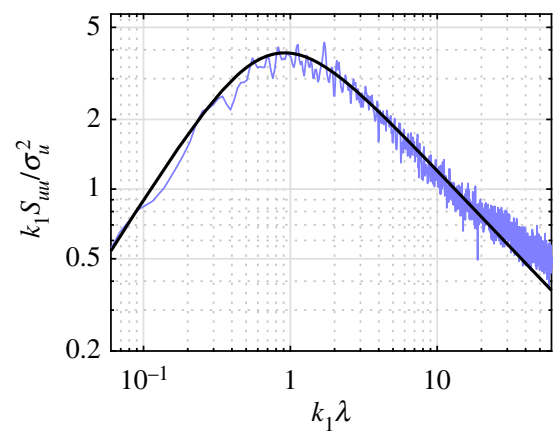

Figure 8. Estimated auto-spectral density (blue line), target spectrum (black line). (Online version in colour.)

lines, and the figures to the right the spectral density. It is observed that the accuracy of the spectral density around the peak increases with increasing total depth covered by the regression lines. However, this happens at the cost of a decreasing accuracy in the high-frequency domain, which is increasingly over-estimated.

A compromise is to keep the base 2, but to increase the number of regression terms to 9 , whereby $j=2^{i-1}=1,2,4,8,16,32,64,128,256$. This set of regression lines contains the previous base 4 set in figure $8 e$, but with an intermediate line between each of those, and retains the fine discretization for small intervals. Simulation results are shown for the spectral density of all three velocity components in figure 9, sampled at the end, the quarter point and the mid-point, marked with blue, magenta and green, respectively. The spectral densities are represented with very good accuracy over the full frequency range. As in the previous example of the square, there is no notable dependence on the sampling point.

The spatial correlations are important characteristics of the simulated field. The correlation coefficients $C_{u u} / \sigma_{u}^{2}, C_{v v} / \sigma_{v}^{2}$ and $C_{w w} / \sigma_{w}^{2}$ are estimated from pairs of sampled records for the points 

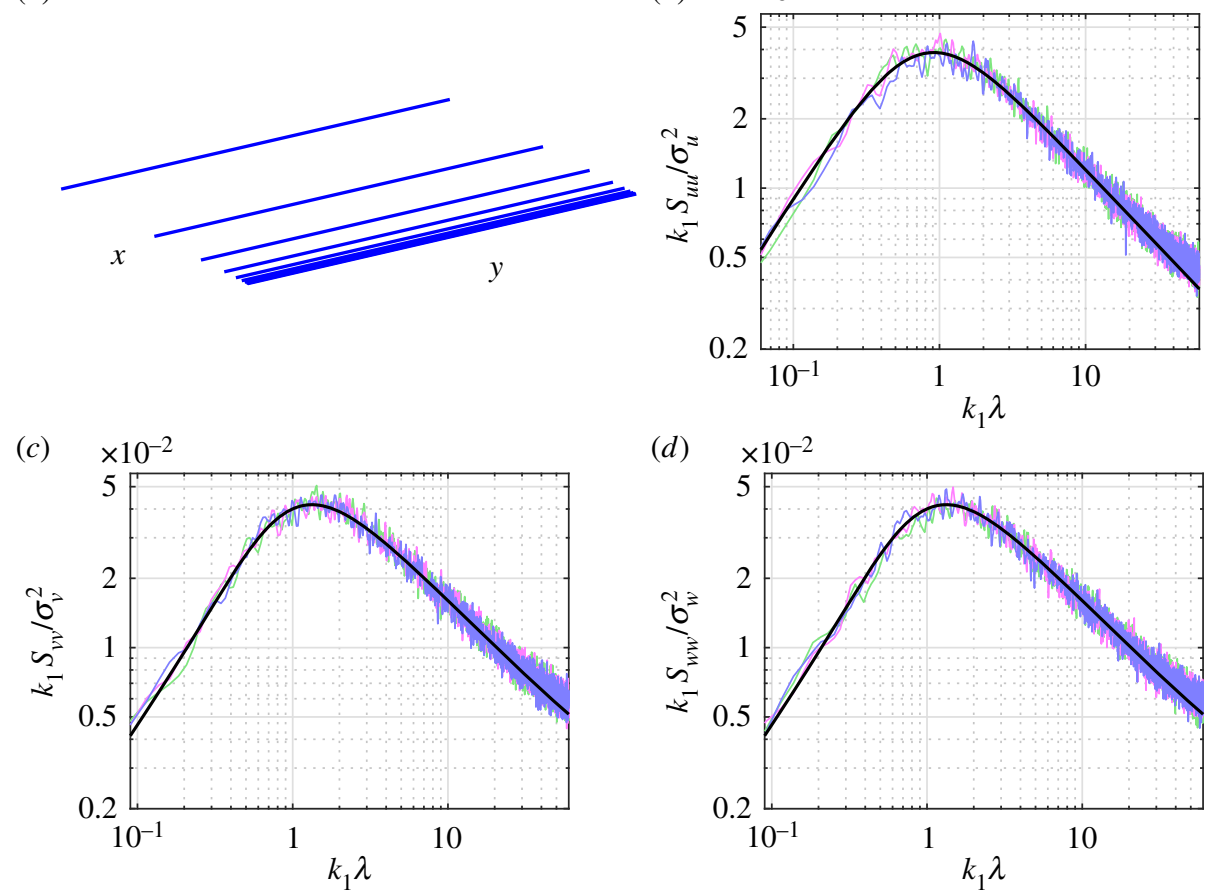

Figure 9. Estimated spectral densities at endpoint (blue line), quarter point (magenta line) and mid-point (green line), target spectrum (black line). (Online version in colour.)

(a)

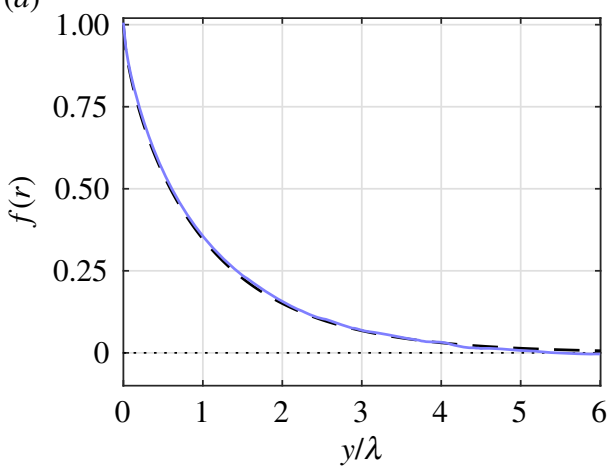

(b)

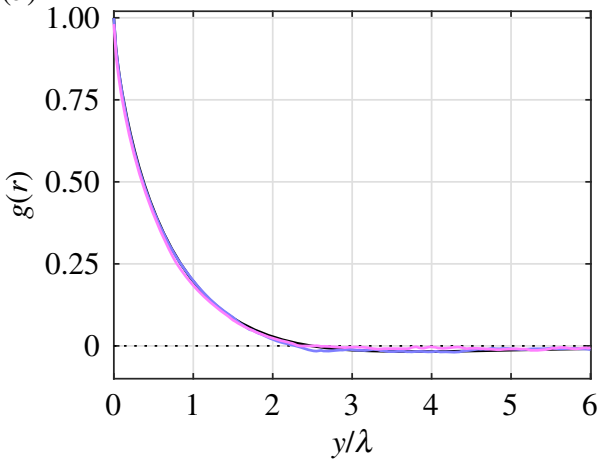

Figure 10. Transverse correlations: (a) $f(r)$ (black line), estimate $C_{v v} / \sigma_{v}^{2}$ (blue line); $(b) g(r)$ (black line), estimate $C_{u u} / \sigma_{u}^{2}$ (blue line) and $C_{w w} / \sigma_{w}^{2}$ (red line). (Online version in colour.)

along the line. The estimated axial and transverse correlations are shown in figure $10 a, b$ together with the theoretical correlation functions $f(r)$ and $g(r)$ from (6.2), respectively. The agreement is seen to be very good over the full range up to $r=6 \lambda$, with no notable difference between the transverse correlation estimates for the along-wind velocity component $u$ and the vertical velocity component $w$.

Finally, the ability of the simulation procedure to resolve the finer details of the turbulent wind velocity field is illustrated by considering the coherence functions $\psi_{11}\left(k_{1} r\right)$ and $\psi_{33}\left(k_{1} r\right)$ for spatial separations $r=0.75 \lambda, 1.5 \lambda, 3.0 \lambda$. The estimated coherence functions $\psi_{11}\left(k_{1} r\right)$ and $\psi_{33}\left(k_{1} r\right)$ 
(a)

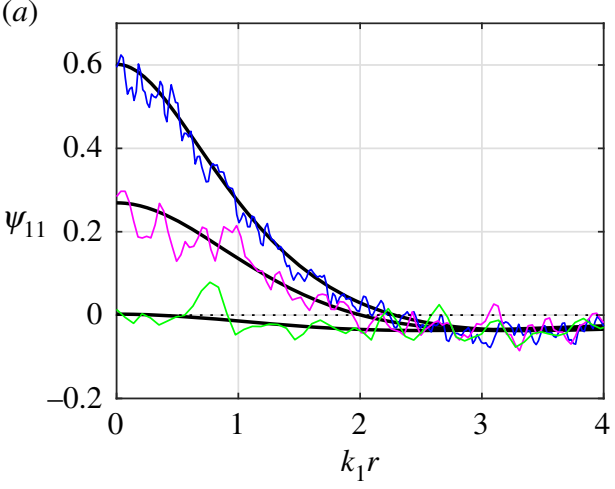

(b)

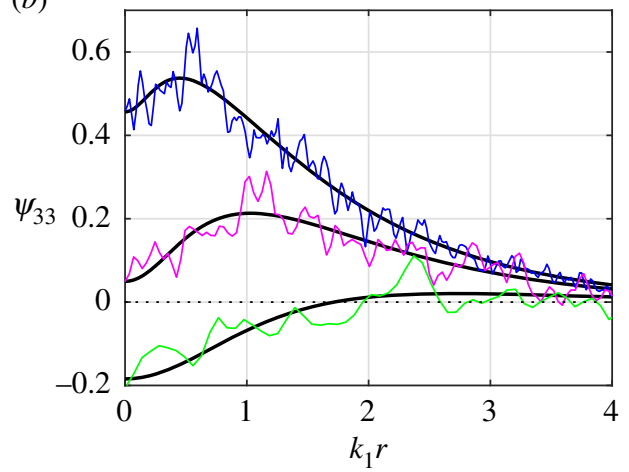

Figure 11. Estimated coherence: $(a) \psi_{11}$, (b) $\psi_{33}$. Separation $0.75 \lambda$ top (blue line), $1.5 \lambda$ middle (magenta line), $3 \lambda$ bottom (green line). Target coherence (black line). (Online version in colour.)

are shown in figures $11 a, b$ together with their theoretical counterparts from (3.14) and (3.21), respectively. In spite of statistical scatter, it is seen that the simulated field captures the underlying coherence structure quite well.

\section{Conclusion}

The theory for isotropic homogeneous turbulence has been summarized, and detailed results presented for both covariances and coherence functions in terms of modified Bessel functions for the case of a generalized von Kàrmàn spectrum, a common representation of the turbulence in natural wind. The typical differences observed in natural wind between the longitudinal, transverse and vertical length scales have been addressed by a simple procedure for generating consistent incompressible orthotropic turbulence by coordinate scaling with corresponding scaling of the velocity component standard deviations.

A procedure is then developed for numerical simulation of a convected turbulent wind field by a modified auto-regressive process, in which transverse regression planes are introduced at exponentially increasing distance from the current transverse plane. The format associated with this procedure is shown to represent the upcoming step of wind velocities as the sum of the conditional mean field and a stochastic part, represented by the conditional covariance matrix. The appropriate simulation matrices are expressed explicitly in terms of these conditional matrices, that in turn are determined explicitly by the homogeneous covariance properties determined in the first part of the paper.

Simulation results with exponentially increasing distance to the regression planes indicate the ability to attain very accurate results for a typical square area of size about one-third of the turbulence length scale and for a line of length six times the length scale, corresponding to a typical wind turbine field and a very long bridge, respectively. The results confirm high accuracy of the simulated frequency spectrum, the transverse correlation and the transverse coherence. Furthermore, the statistics of the simulated records appear to be independent of the particular points chosen in the cross-section. The sequential nature of the procedure and the rather free selection of simulation points in the cross-section plane make it highly competitive with Fourier-based spectral methods.

Data accessibility. This article has no additional data.

Authors' contributions. General theoretical results and the basic form of the simulation procedure were developed by S.K. Simulation code and examples were developed by R.N.M. Both S.K. and R.N.M. contributed to writing the final paper.

Competing interests. We declare we have no competing interests.

Funding. Partial support for RNM from Innovation Fund Denmark. 
Acknowledgements. The paper is part of the project Aerodynamic Stability of Long Span Bridges, sponsored jointly by Innovation Fund Denmark, Rambøll and the Technical University of Denmark.

\section{References}

1. Shinozuka M, Deodatis G. 1996 Simulation of multidimensional Gaussian stochastic fields by spectral representation. Appl. Mech. Rev. 49, 29-53. (doi:10.1115/1.3101883)

2. Deodatis G. 1996 Simulation of ergodic multivariate stochastic processes. J. Eng. Mech. 122, 778-787. (doi:10.1061/(ASCE)0733-9399(1996)122:8(778))

3. Batchelor GK. 1953 The theory of homogeneous turbulence. Cambridge, UK: Cambridge University Press.

4. Mann J, Krenk S. 1994 Fourier simulation of non-isotropic wind field model. Proceedings of ICOSSAR '93, Innsbruck, Austria, August 9-13, 1993, vol. 3 (eds GI Schueller, M Shinozuka, JTP Yao), pp. 1669-1674. Rotterdam: Balkema.

5. Mann J. 1998 Wind field simulation. Probab. Eng. Mech. 13, 269-282. (doi:10.1016/S0266-8920(97)00036-2)

6. Li Y, Kareem A. 1995 Stochastic decomposition and application to probabilistic dynamics. J. Eng. Mech. 119, 162-174. (doi:10.1061/(ASCE)0733-9399(1995)121:1(162))

7. Di Paola M. 1998 Digital simulation of wind field velocity. J. Wind Eng. Ind. Aerodyn. 74-76, 91-109. (doi:10.1016/S0167-6105(98)00008-7)

8. Di Paola M, Gullo I. 2001 Digital generation of multivariate wind field processes. Probab. Eng. Mech. 16, 1-10. (doi:10.1016/S0266-8920(99)00032-6)

9. Chen X, Kareem A. 2005 Proper orthogonal decomposition-based modeling, analysis, and simulation of dynamic wind load effects on structures. J. Eng. Mech. 131, 325-339. (doi:10.1061/(ASCE)0733-9399(2005)131:4(325))

10. Solari G, Carassale L, Tubino F. 2007 Proper orthogonal decomposition in wind engineering. Part 1: a state-of-the-art and some prospects. Wind Struct. 10, 153-176. (doi:10.12989/ was.2007.10.2.153)

11. Carassale L, Solari G, Tubino F. 2007 Proper orthogonal decomposition in wind engineering. Part 2: theoretical aspects and some applications. Wind Struct. 10, 177-208. (doi:10.12989/ was.2007.10.2.177)

12. Stefanou G, Papadrakakis M. 2007 Assessment of spectral representation and KarhunenLoève expansion methods for the simulation of Gaussian stochastic fields. Comput. Methods Appl. Mech. Eng. 196, 2465-2477. (doi:10.1016/j.cma.2007.01.009)

13. Hémon P, Santi F. 2007 Simulation of a spatially correlated turbulent velocity field using biorthogonal decomposition. J. Wind Eng. Ind. Aerodyn. 95, 21-29. (doi:10.1016/j.jweia. 2006.04.003)

14. Davenport AG. 1977 The prediction of the response of structures to gusty wind, In Safety of structures under dynamic loading (eds I Holand, D Kavlie, G Moe, R Sigbjörnsson), pp. 257-284. Trondheim, Norway: Tapir.

15. Yang WW, Chang TYP, Chang CC. 1997 An efficient wind field simulation technique for bridges. J. Wind Eng. Ind. Aerodyn. 67-68, 697-708. (doi:10.1016/S0167-6105(97)00111-6)

16. Cao Y, Xiang H, Zhou Y. 2000 Simulation of stochastic wind velocity field of long-span bridges. J. Eng. Mech. 126, 1-6. (doi:10.1061/(ASCE)0733-9399(2000)126:1(1))

17. Huang G, Liao H, Li M. 2013 New formulation of Cholosky decomposition and applications in stochastic simulation. Probab. Eng. Mech. 34, 40-47. (doi:10.1016/j.probengmech.2013.04.003)

18. Peng L, Huang G, Kareem A, Li Y. 2013 An efficient space-time based simulation approach of wind velocity field with embedded conditional interpolation for unevenly spaced locations. Probab. Eng. Mech. 34, 40-47. (doi:10.1016/j.probengmech.2013.04.003)

19. Benowitz BA, Deodatis G. 2015 Simulation of wind velocity on long span structures: a novel stochastic wave based model. J. Wind Eng. Ind. Aerodyn. 147, 154-163. (doi:10.1016/j.jweia.2015.10.004)

20. Li Y, Hu P, Xu X, Qiu J. 2017 Wind characteristics at bridge site in a deep-cutting gorge by wind tunnel tests. J. Wind Eng. Ind. Aerodyn. 160, 30-46. (doi:10.1016/j.jweia.2016.11.002)

21. Peng Y, Wang S, Li J. 2018 Field measurement and investigation of spatial coherence for near-surface strong winds in southeast China. J. Wind Eng. Ind. Aerodyn. 172, 423-440. (doi:10.1016/j.jweia.2017.11.012) 
22. Fenerci A, Øiseth O. 2018 Strong wind characteristics and dynamic response of a long-span suspension bridge during a storm. J. Wind Eng. Ind. Aerodyn. 172, 116-138. (doi:10.1016/j.jweia.2017.10.030)

23. Krenk S. 1996 Wind field coherence and dynamic wind forces. IUTAM symposium on advances in nonlinear stochastic mechanics (eds A Naess, S Krenk), pp. 269-278. Dordrecht, The Netherlands: Kluwer.

24. Kristensen L, Jensen NO. 1979 Lateral coherence in isotropic turbulence and in the natural wind. Bound. Layer Meteorol. 17, 353-373. (doi:10.1007/BF00117924)

25. von Kàrmàn T. 1948 Progress in the statistical theory of turbulence. Proc. Natl Acad. Sci. USA 34, 530-539. (doi:10.1073/pnas.34.11.530)

26. Mignolet MP, Spanos PD. 1992 Simulation of homogeneous two-dimensional random fields: part I - AR and ARMA models. J. Appl. Mech. 59, 260-269. (doi:10.1115/1.2899499)

27. Spanos PD, Mignolet MP. 1992 Simulation of homogeneous two-dimensional random fields: part II - MA and ARMA models. J. Appl. Mech. 59, 270-277. (doi:10.1115/1.2899500)

28. Di Paola M, Zingales M. 2008 Stochastic differential calculus for wind-exposed structures with auto-regressive continuous (ARC) filters. J. Wind Eng. Ind. Aerodyn. 96, 2403-2417. (doi:10.1016/j.jweia.2008.04.003)

29. Kareem A. 2008 Numerical simulation of wind effects: a probabilistic perspective. J. Wind Eng. Ind. Aerodyn. 96, 1472-1497. (doi:10.1016/j.jweia.2008.02.048)

30. Krenk S. 2011 Explicit calibration and simulation of stochastic fields by low-order ARMA processes. COMPDYN 2011. ECCOMAS thematic conference on computational methods in structural dynamics and earthquake engineering (eds M Papadrakakis, M Fragiadakis, V Plevris), paper 550, pp. 1-10. Barcelona, Spain: ECCOMAS.

31. Møller RN, Krenk S, Svendsen MN. Submitted Turbulent wind response of long-span bridges including flutter. Department of Mechanical Engineering, Technical University of Denmark.

32. Olver FWJ, Lozier DW, Boisvert RF, Clark CW. 2010 NIST handbook of mathematical functions. Cambridge, UK: Cambridge University Press. 